\title{
Historia de la Universidad Distrital: capítulo: la fundación (1948-1950) ${ }^{1}$ History of the university district: chapter: the foundation (1948-1950) História da universidade do distrito: capítulo: a fundação (1948-1950)
}

Recibido: octubre de 2012

Aceptado: diciembre de 2012
Carlos Arturo Reina Rodríguez ${ }^{2}$

\section{Resumen}

La Universidad Distrital Francisco José de Caldas cuenta con más de 60 años de existencia. Su fundación es el resultado de un proceso histórico acumulado por la experiencia de las Escuelas de Artes y Oficios y las Escuelas Industriales en el país, creadas en el siglo XIX. La necesidad de formar a los sectores pobres que no podían acceder a la educación superior fue una de las razones de su existencia. En la constitución del Colegio Municipal de Bogotá en 1948 se destacaron personajes como Antonio García Nossa, Gabriel Anzola o Jorge Eliécer Gaitán. Hacia 1850, el 6 de agosto y bajo la rectoría del presbítero Daniel de Cayzedo, fue fundada la Universidad Municipal de Bogotá Francisco José de Caldas. Esta investigación busca brindar nuevos referentes para la construcción de una historia de la Universidad Distrital y aportar elementos para la historia de la Educación Superior en Colombia.

Palabras clave: historia de Bogotá, escuelas industriales, Universidad Distrital, educación superior.

\begin{abstract}
The University District "Francisco José de Caldas", has over 60 years of existence. Its foundation is the result of a historical process experience accumulated by the School of Arts and Crafts and Industrial Schools in the country, created in the nineteenth century. The need to train the poor who could not access higher education, was one of the reasons for its existence. In the constitution of Bogota Municipal College in 1948, she stood out as Antonio Garcia Nossa characters, Gabriel Anzola or Jorge Eliezer Gaitan. By 1850, the August 6 and under the guidance of the priest Daniel Cayzedo was founded Bogota City University "Francisco José de Caldas". This research aims to provide new standards for the construction of a history of the University District and to inform the history of Higher Education in Colombia
\end{abstract}

Keywords: history of Bogota, industrial schools, University District, higher education.

1 Artículo de revisión.

2 Doctor en Historia especializado en Historia Cultural, Universidad Nacional de Colombia. Docente de la Facultad de Ingeniería, Ingeniería de Sistemas, Universidad Distrital. Contacto: careina@udistrital.edu.co 


\section{Resumo}

A Universidade Distrital "Francisco José de Caldas", tem mais de 60 anos de existência. Sua fundação é o resultado de um processo histórico experiência acumulada pela Escola de Artes e Ofícios e escolas industriais no país, criados no século XIX. A necessidade de treinar os pobres que não podiam acessar o ensino superior, foi uma das razões para a sua existência. Na constituição de Bogotá Municipal College, em 1948, ela se destacou como Antonio Garcia Nossa, Gabriel Anzola ou Jorge Eliezer Gaitan. Em 1850, a 06 de agosto e, sob a orientação do padre Daniel Cayzedo foi fundada Bogotá City University "Francisco José de Caldas". Esta pesquisa tem como objetivo proporcionar novos padrões para a construção de uma história do bairro da universidade e informar a história da Educação Superior na Colômbia

Palavras chave: história de Bogotá, escolas industriais, University District, o ensino superior.

\section{Introducción}

La Universidad como institución ha tenido como responsabilidad la formación de las élites intelectuales que han contribuido al desarrollo social, político, económico y cultural del país. Los diferentes momentos desde su creación la hacen ver como una institución en permanente crisis con periodos más o menos importantes, que dependieron de la coyuntura social y política del momento, caracterizada por el predominio hegemónico de partidos políticos o coaliciones de estos. Por otra parte, los estudios sobre el tema nos muestran una institución muy ligada a los periodos políticos que han caracterizado la historia del país y de Bogotá. Por esta razón, la mayoría de los trabajos relacionados clasifican los periodos de evolución de la universidad colombiana respecto al desarrollo político, económico y social de la sociedad colombiana.

Después de la Guerra de los Mil Días, prácticamente el país quedó devastado. Se vio la necesidad de reorganizar buena parte de las estructuras de la sociedad, donde el aparato productivo fue parte fundamental. La creación de Escuelas de Artes y Oficios destinadas a los sectores más pobres se convirtió en una política que buscó ocupar a los cientos de desempleados y convertirlos en mano de obra útil y autogestionable para evitar y controlar la delincuencia así como la vagancia. Estas Escuelas de Artes y Oficios, así como las Escuelas
Industriales que se crearon bajo la tutoría de los colegios existentes en la ciudad, dieron como resultado la aparición de proyectos para la creación de "universidades para los pobres", uno de los cuales sería a la postre Universidad Municipal de Bogotá, hoy Universidad Distrital Francisco José de Caldas. Este trabajo intenta reconstruir los primeros años de vida universitaria de nuestra alma mater, revisar el proceso de creación hasta la aparición como Universidad, así como también el de su primera facultad: la Facultad de Ingeniería. Este es un primer ejercicio hacia la construcción de una historia institucional pero también cultural a la cual se han vinculado, en distintos momentos, estudiantes del proyecto curricular de Ingeniería de Sistemas.

En el campo de Historia de la Educación se ha tejido un amplio y fructífero campo de información y producción conceptual, en el ámbito tanto de la educación colombiana como de la latinoamericana. De ello dan cuenta, por ejemplo, los estudios en esta materia que adelantan la Universidad Pedagógica y Tecnológica de Tunja, a través del Doctorado en Historia de la Educación, así como el Doctorado en Educación desarrollado en la misma Universidad Distrital.

Como parte de la reconstrucción de la historia de la Universidad Distrital, se ha avanzado sobre la búsqueda de fuentes secundarias y primarias. En cuanto a estas últimas, los resultados indican un 
número limitado de documentos al respecto. El balance historiográfico de Blanca Ortiz titulado "Aproximación a la historiografía y fuentes de la Universidad Francisco José de Caldas”, publicado en la Revista Historia de la Universidad Colombiana (1998). En ella se hace un balance de los documentos históricos existentes al respecto y también se señalan la ausencia de numerosos archivos la dificultad para acceder al llamado archivo "muerto" de la Universidad. Se destaca el libro de Rafael Díaz Borbón, La Universidad Distrital: ¿paradigma de la crisis y disolución de la Universidad Pública? (1997), donde se examina la condición de la Universidad Distrital en un análisis comparativo con otras universidades, pretendiendo mostrar cómo la Universidad Distrital es reflejo a lo largo de su historia de lo que acontece en las universidades públicas del país.

También está la tesis monográfica de Ramírez y Silva "Elementos para una historia institucional de la Universidad Distrital" (1988), que analiza de manera crítica el periodo entre 1948 y 1987. Así mismo el periódico Signos, número 9 de agosto de 1997, donde se presentó una reseña histórica de la Universidad, que privilegia los aspectos organizativos y de infraestructura. También se encuentran los libros 60 años de Memoria y Vida (2008) y En pos de la memoria: fuente para la historia de la Universidad Distrital Francisco José de Caldas(2011), editados por Flor Alba Santamaría (2008). Otros documentos son los de Reina (2008) y Jiménez \& Guevara (2001).

En cuanto a las fuentes primarias, el trabajo investigativo se apoya en la búsqueda de material correspondiente al periodo en estudio. Para ello, han servido de fuentes documentales los archivos correspondientes a los Anales del Concejo de Bogotá, las actas municipales de la época, los registros fotográficos y las publicaciones en periódicos de la época, como El Tiempo, El Espectador y El Siglo. Al mismo tiempo se han recogido entrevistas con algunos de los primeros estudiantes que se vincularon a la Universidad, así como también se ha revisado el material existente en los archivos y bibliotecas de la ciudad en pos de reconstruir el contexto de creación de la fundación de la Universidad.
Uno de los paradigmas de la mayor parte de estos documentos, así como de los existentes en el portal web de la Universidad Distrital, señala que la universidad fue fundada por iniciativa del presbítero Daniel de Cayzedo. Así mismo, que el nombre de la misma, "Francisco José de Caldas", se obtuvo años después de su fundación. Este trabajo pretende mostrar que el presbítero Cayzedo no fue el fundador del Colegio Municipal y que solo se integró a él hasta 1950, dos años después de creado. Que tampoco fue el primer rector y que existieron otros personajes, como Antonio García Nossa, que la historia tradicional no menciona pero que fueron los mentores tanto del colegio como de la Universidad Municipal. El objetivo fundamental tiene que ver con el aporte para corregir la narración histórica del origen y fundación de la Universidad Distrital $y$, de esta manera, para reconocer las raíces de la misma.

\section{Materiales y métodos}

Para el desarrollo de esta etapa se tomaron documentos secundarios que tampoco ilustraban respecto a la fundación de la Universidad, sino que, por el contrario, retomaban los argumentos de origen en la persona del presbítero Daniel de Cayzedo. Tampoco aportaron significativamente los documentos existentes en torno a Antonio García Nossa, ni los que hacían referencia a Jorge Eliécer Gaitán. Sus escritos, autobiografías o entrevistas, no mencionan al Colegio Municipal ni mucho menos a la Universidad Municipal de Bogotá. Ellos apenas dejan intuir el interés por la educación industrial pero no concluyen en el objetivo de crear colegio o universidad alguna. De allí que la reconstrucción de la información tuvo que hacerse a partir de la revisión de documentos primarios, tales como los Anales del Concejo de Bogotá, los Registros Municipales y la búsqueda de información de las primeras publicaciones del Colegio y la Universidad Municipal. Es importante anotar que la Universidad no cuenta con un archivo histórico de la misma y que los documentos existentes se encuentran de manera fragmentada en algunas dependencias de esta y que, respecto a los primeros años, la información es escasa. 
En el transcurso de presentación de este documento se logró establecer que entre 1948 y 1951 el Colegio Municipal publicó tres libros editados por la Imprenta Municipal. El primero, de 1948, narraba el origen del proyecto y los autores del mismo. Los dos restantes forman parte de un compilado de escritos en prosa y verso de contenido literario, de autoría de los profesores del plantel. Además se editó una revista. No obstante, el referente apenas pasaba de contar con el índice. La búsqueda en el Archivo General de la Nación, el Archivo de Bogotá, las Bibliotecas Nacional y Luis Ángel Arango reportó resultados negativos. A través de la búsqueda por la Web se encontró un referente que apenas señalaba la existencia de tales documentos. El lugar de ubicación, después de una búsqueda exhaustiva, fue la Library of Congress, Biblioteca del Congreso de los Estados Unidos, con quienes se tramitó el envío de los documentos. Finalmente fueron remitidos a través de vía electrónica, con lo cual una vez realizada la revisión preliminar se pudo constatar lo que hasta entonces era una hipótesis: que el cerebro tras la fundación del Colegio era en realidad el economista bogotano Antonio García Nossa y que el primer rector del Colegio Municipal había sido Gabriel Anzola Gómez.

\section{Resultados}

A partir del análisis de documentos mencionada antes, se estableció un estudio de carácter histórico que dio como resultado:

\section{La importancia de las Escuelas Industriales, de Artes y Oficios}

A partir de1850, en medio del debate de la existencia de la Universidad por parte de las facciones que definían las líneas del partido liberal y del partido conservador, se empezó a pensar en la necesidad de crear Escuelas de Artes y Oficios, para que se formaran los sectores pobres del país. La primera Escuela de Artes y Oficios fue creada mediante la Ley 22 del 22 de septiembre de 1867, al tiempo que la Universidad Nacional, pero no pudo iniciar debido a la falta de espacios físicos y de presupuesto. Por tanto esta escuela terminó a la postre siendo asimilada por la Universidad Nacional como parte de una de sus facultades.

A principios del siglo XX, solo había el bachillerato clásico conocido con el nombre de Filosofía y Letras, el cual era el único aceptado para el ingreso a las universidades. El 26 de octubre de 1903 fue una fecha importante en el desarrollo de la enseñanza industrial en Colombia porque el presidente José Manuel Marroquín sancionó la Ley 39 de 1903. Esta ley dividió la enseñanza oficial en primaria, secundaria, profesional, industrial y artística, en la cual quedaron facultadas las asambleas para fundar y sostener escuelas de este tipo en la capital de cada departamento, y además en las provincias que estimaran convenientes, en las cuales se enseñaran artes manufactureras y especialmente el manejo de máquinas aplicables a las pequeñas industrias.

Estas entidades administrativas pudieron entonces crear y sostener en cada una de las cabeceras de provincia un taller para la enseñanza gratuita de un arte u oficio por lo menos, según las necesidades y las condiciones de cada localidad. También los gobernadores de los departamentos quedaron facultados para dictar los reglamentos de las escuelas y talleres, bajo la supervisión y el visto de aprobación del gobierno central. Esa misma norma restableció la Escuela de Minas de Medellín, que tuvo por objeto formar ingenieros científicos y prácticos capaces de dirigir la exploración y explotación de minas. Igualmente se autorizó para que el gobierno apoyara la fundación y sostenimiento en Bogotá del Instituto de San Antonio, en el cual se debían ofrecer cursos y enseñanza teórica y práctica de agricultura y artes y oficios mecánicos, especialmente para los niños pobres.

El 19 de marzo de 1904, Antonio José Uribe, junto con el hermano visitador provincial de los Hermanos de las Escuelas Cristianas, inauguró la Escuela Central de Artes y Oficios de Bogotá. Al año siguiente se expidió el Decreto 146 por el cual se reorganizó la escuela y se estableció el plan de estudios con las especialidades de mecánica, fundición, herrería, calderería, ebanistería, zapatería, talla, modelaje y fabricación de tejidos. Los progresos del Asilo fueron motivos para convertirlo en Escuela Central de Artes y Oficios. En 1916, el 
presidente José Vicente Concha y el ministro de Educación Pública Emilio Ferrero decretaron que la escuela podía otorgar los títulos de ingeniero en Electricidad y Artes Mecánicas, ingeniero en Electricidad e Industrias Textiles e ingeniero en Electricidad y Arte Industrial Decorativo. Esta fue la primera Universidad Industrial de Colombia a pesar de que en 1919 el presidente Marco Fidel Suárez, a través del Decreto 721 del 4 de abril, cambió el nombre de escuela por el de Instituto Técnico Central.

En 1931, mediante el Decreto 2219 del 18 de diciembre, el gobierno nacional fusionó el Instituto Técnico Central con la Facultad de Ingeniería de la Universidad Nacional y fueron deslazados los hermanos cristianos del Técnico Central. Para 1937 empezó a funcionar en el edificio del instituto la Escuela Industrial de Bogotá, que fue la sucesora del Técnico Central hasta 1951 y se convirtió en la única institución de la ciudad en ofrecer carreras técnicas. Su declive se inició, tras nutridas difamaciones y denuncias de corrupción, a partir de 1942.El departamento politécnico del Colegio Municipal de Bogotá (UD) vino a ser el lugar donde se dio continuación con las escuelas industriales siguiendo la normatividad antes señalada.

También fue en 1904 cuando el ministro de Instrucción Pública Antonio José Uribe logró, junto con la comunidad salesiana, que se aprobara un nuevo tipo de bachillerato. Lo llamaron "moderno" y su pensum no contenía latín ni griego. Posteriormente el Dr. Emilio Ferrero, ministro de Instrucción Pública, dictó la Resolución 23 del 13 de junio de 1916, donde se hizo extensivo a otros tipos de bachillerato y, con ello, abrió posibilidades de obtención de grados bajo otras modalidades. El bachillerato moderno del que hablaron los padres salesianos incorporó al bachillerato clásico áreas como Ciencias Físicas y Químicas, Ciencias Biológicas, Lenguas Vivas y Comercio. Dentro de este proceso el Asilo San José, establecimiento oficial, se transformó en el Instituto Técnico Central, inaugurado el 19 de marzo de 1904.

La necesidad de ampliar la cobertura educativa se confirmó con los resultados de los censos, en particular el de 1938 que mostró que había 1.817.312 niños en edad escolar -de 7 a 14 años-y, de ellos, solamente 627.730 asistían a la escuela primaria (Censo general de Población 1942, p.138).El aumento de la cobertura y la disminución de las tasas de analfabetismo se convirtieron en prioridad de los gobiernos del siglo XX, lo que se evidenció en un aumento en las tasas de escolaridad reflejadas en el promedio de acceso a la educación, tanto en las poblaciones urbanas como en las rurales, con un distanciamiento significativo entre la primera respecto de la segunda.

En el estudio que realizó la economista Carmen Flórez (2000) acerca del desarrollo sociohistórico de la población colombiana a lo largo del siglo XX, encontró que la población de menores de 15 años, que a principios del siglo representaba el 40,6\%, aumentó a $45,3 \%$ en 1964 , y se redujo a $34,5 \%$ en 1993. Este comportamiento de la población joven implicó una evolución contraria en la población entre 15 y 65 años; inicialmente su participación disminuyó de 56,3\% a 52\% entre 1905 y 1964, para luego aumentar nuevamente hasta llegar a representar el $61 \%$ de la población total de 1993. Esa variación se reflejó en el aumento de la presencia de la juventud en los años sesenta y con ello de la vida y la cultura universitaria en el país.

La escolaridad primaria cumplió la función primordial de ampliar la base de población que sabía leer y escribir, especialmente en las áreas rurales. Mientras tanto, en el sector de la educación secundaria, hasta 1948, fue privilegiada por la fundación de colegios de carácter privado como el Gimnasio Moderno o el Liceo Cervantes, además de otros de pequeño calado, impulsados por nobles maestros que, pese a su interés por ampliar la cobertura educativa, debían cobrar por ello, lo cual excluía a la mayoría de la población. Además, en Bogotá estaban los colegios nacionales San Bartolomé, Nicolás Esguerra y Camilo Torres. La ciudad no contó con ninguno propio hasta la fundación del Colegio Municipal de Bogotá en 1948.

En cuanto al acceso a la educación superior, era más restringido ya que la oferta tampoco era muy amplia, y esta se dividía entre las ofertas de las universidades privadas como el Externado, el Rosario, la Libre y la Javeriana, o la que proveían la Escuela 
Normal Superior y la Universidad Nacional de Colombia. Quienes no podían vincularse a ninguna de estas instituciones contaron con la oferta de las Escuelas de Artes y Oficios y las Escuelas Industriales, consideradas como instituciones de cursos menores. Con las reformas de los años treinta, impulsadas por los gobiernos liberales, se abrieron las puertas a campos de formación más amplios que marcaron la creación de carreras que tuvieron que ver con el mundo de la administración, negocios y economía, ingenierías especializadas en procesos industriales y la pedagogía. Con el impulso del proceso de industrialización creció la clase obrera cuyos hijos se encontraron ante el problema de la formación técnica para el trabajo en una ciudad cada vez más grande y más poblada, donde las posibilidades de empleo se reducían.

Ante la desaparición del ITC y el declive y posterior desaparición de la Escuela Industrial de Bogotá, la ciudad quedó desprovista de un lugar de formación para los hijos de los obreros bogotanos.De manera indirecta, el Departamento Politécnico del Colegio Municipal de Bogotá se convirtió en el heredero de los logros y las luchas de las Escuela de Artes y Oficios de Bogotá, así como del Instituto Técnico Central y de la Escuela Industrial de Bogotá.

\section{El papel de Jorge Eliécer Gaitán y el Colegio Municipal}

Se dice que Jorge Eliécer Gaitán tuvo que ver con el origen del Colegio Municipal de Bogotá y con la Universidad Municipal. Lo concreto es que en ningún documento revisado Gaitán se refirió a alguna propuesta para crear institución alguna. Más bien señaló el interés por apoyar la enseñanza industrial, lo cual, interpretado en conjunto con la presencia de su amigo Antonio García Nossa, señalaría que su incidencia en la creación del Colegio Municipal en 1948 está tácitamente presente en el proyecto que García dirigió al Concejo Municipal en diciembre de 1947.

Durante el paso de Gaitán por el Ministerio de Educación creó un Consejo Técnico de la Enseñanza Primaria, el Consejo Nacional de Segunda Enseñan $\neg$ za, y dio un impulso para que se incorporara la enseñanza agraria en las escuelas y colegios rurales y urbanos. Promovió la construcción y creación de más de 50 instituciones escolares de primaria, así como el fortale $\neg$ cimiento de la enseñanza comercial, el ordenamiento de la carrera administrativa y la creación de un instituto para el desarrollo científico denominado Ateneo Nacional de Altos Estudios.

En 1940, Gaitán criticó que el nombre de escuelas industriales fuera usado para referirse a diversas formas de educación que no quedaban claras hasta ese momento, por lo que se hacía necesaria una reforma donde se establecieran las diferencias en ese tipo de educación. La descripción realizada por Gaitán en la época da cuenta de los tipos de escuelas existentes en ese momento:

En la actualidad, como primera etapa de la enseñanza industrial existen, aun en forma muy deficiente y escasa, las llamadas escuelas complementarias que son una especie de combinación entre la primaria y la escuela de oficios propiamente dicha. Esta forma de solución obligada por los limitados recursos de que disponen la enseñanza industrial y la primaria, tiene, sin embargo, el defecto de no llenar ninguna de las dos necesidades en forma completa, pues que ni está en capacidad de desarrollar metódicamente su pensum estrictamente intelectual, que es, lo repito, de escuela primaria, ni de poder dotar suficientemente de los conocimientos de labores manuales, ya que si bien es cierto se dan algunas nociones sobre oficios, no bien estudiados y orientados según las necesidades del medio, falta el tiempo, que se lleva la simple educación primaria, para el apropiado desarrollo del oficio que tiende a formar el obrero calificado (Gaitán,1940, p.290).

En el Informe Gaitán de 1947, escrito en compañía de Antonio García Nossa, afirma que, debido a las deficiencias en primaria, el desarrollo dela segunda etapa de formación era igualmente pobre, por lo que se hacía necesario realizar una reforma al sistema de organización y enseñanza industrial que fuera acorde con la realidad. Su propuesta fue la siguiente: 
$1^{\circ}$ Escuelas elementales rotatorias. (Para los alumnos de las escuelas primarias).

$2^{\circ}$ Escuelas medias de oficios. (Para obreros calificados).

$3^{\circ}$ Escuelas secundarias. (Para oficiales expertos).

$4^{\circ}$ Institutos superiores. (Para técnicosindustriales).

Aquí se destacó el interés por diversificar el bachillerato clásico y abrir otras opciones de formación distintas dirigidas hacia un bachillerato técnico a partir del cuarto año de secundaria. "Es indispensable que la intensificación de ciertas ramas de la ciencia se haga sentir como educación secundaria, según la carrera profesional en perspectiva, y que al bachillerato estrictamente clásico corresponda la existencia del bachillerato técnico, según las diversas profesiones” (Gaitán, 1940, p. 296).

Gaitán fue un defensor de la educación técnica en el país. Sus ideas quedaron plasmadas en la propuesta de 1947 presentada por el concejal liberal Antonio García Nossa ante el Concejo de Bogotá. A pesar de que se presume la influencia de Gaitán en la fundación del Colegio Municipal de Bogotá, esta no quedó plasmada bajo su firma. La fundación de esta institución se puede enmarcar dentro de los ideales del gaitanismo, aunque no necesariamente como uno de sus proyectos.

\section{Las características políticas y educativas a fines de la década de los cuarenta en relación con la creación del Colegio Municipal}

Un aspecto importante a tener en cuenta fue el escenario político de la época. En 1945, los liberales perdieron las elecciones ante el candidato conservador Mariano Ospina Pérez. La división del liberalismo entre Gabriel Turbay y Jorge Eliécer Gaitán cobró la pérdida del poder. Aun así, en las elecciones municipales de 1947 los liberales se quedaron con la mayoría de las plazas en Bogotá. Esto permitió que muchos proyectos fueran aprobados durante su legislatura y que entre ellas estuviera la creación del Colegio Municipal en febrero de 1948, así como la creación del Departamento Politécnico en julio del mismo año. De hecho, el
Colegio Municipal de Bogotá Jorge Eliécer Gaitán, nombre que adoptó luego del asesinato del líder liberal, fue el primero que contó con la modalidad de bachillerato técnico y con un departamento técnico en la ciudad.

En poco menos de cinco años, el panorama de la educación superior en Bogotá y el país tomó otro rumbo. En 1945 fue nombrado como rector de la Universidad Nacional el ideólogo liberal Gerardo Molina, quien abrió los debates en torno a la autonomía universitaria e impulsó la creación de varias carreras profesionales. A pesar de ello, tuvo que soportar la huelga estudiantil de 1947 que lo llevó a su posterior retiro de la rectoría. También se consolidó el Instituto Caro y Cuervo y aparecieron la Universidad Industrial de Santander, cuyas actividades iniciaron el primero de marzo de 1948 como una Facultad de Ingeniería dentro de las instalaciones del Instituto Técnico Superior Dámaso Zapata, y la Universidad del Tolima en 1945, a pesar de que inició labores solo 10 años después. En 1948 aparecieron universidades privadas como la Universidad de los Andes y en 1950 la Universidad de Medellín y la Universidad Municipal de Bogotá.

Al mismo tiempo, en 1947, se llevó a cabo la reforma de la educación pública donde la dirección de educación fue dividida en cinco departamentos: departa $\neg$ mento técnico, administrativo, de negocios generales, de medicina escolar y de construcciones escolares. Tambiénse debatió acerca de la necesidad de una reforma para la educación universitaria, mientras se escuchaban los consejos del ingeniero electrónico Italo Amore en torno a la necesidad de formar profesionales en la electrónica. (El Tiempo, marzo de 1947, p. 15).En 1949 hubo pronunciamientos oficiales frente a la necesidad de incentivar la educación industrial:

La enseñanza industrial ha sido una de las hijas pobres del Estado, la última en aparecer en el escenario educativo, no bien comprendida y aprobada, y por lo mismo con mínimos recursos. Por desgracia para nuestro país, el trabajo técnico ha sido subestimado en épocas anteriores, Por esto la enseñanza técnica ha sido la última en aparecer en las preocupaciones del gobierno... (El Siglo, $10 \mathrm{de}$ abril de 1949, p. 2). 
De acuerdo con el gobierno, solo existían 14 escuelas industriales en todo el país. Por esta razón, el ministro de Educación Norberto Solano expresó en abril del mismo año:

El gobierno está vivamente interesado por modificar los sistemas educativos que hasta hoy se han practicado en Colombia y para efecto Colombia necesita más técnicos que bachilleres en potencia, más escuelas industriales y agrícolas que colegios de segunda enseñanza.... entre ser médico o técnico mecánico, entre la abogacía y la técnica eléctrica, los jóvenes, nuestros jóvenes escogen lo primero, porque tienen un concepto equivocado, peyorativo del trabajo manual...es impostergable llevar este beneficio a todos los sectores del personal que ocupa la industria nacional. Desde el modesto obrero hasta el ingeniero técnico( $E l$ Siglo,10 de abril de 1949, p. 2).

La clasificación propuesta por el gobierno, amparado en la Ley 143 para la Reorganización y Fomento de la Educación Técnica, planteó:

a) Preparación de obreros no calificados.

b) Perfeccionamiento de los obreros calificados.

c) Formación y perfeccionamiento de artesanos especializados y con conocimientos técnicos, en todas las actividades que reclama el país según el medio y las circunstancias del ambiente y materias primas.

d) Perfeccionamiento de artesanos empíricos.

e) Formación de expertos para la industria.

f) Formación de técnicos que se responsabilicen y estén en capacidad de resolver los problemas más concretos de los talleres de la industria.

g) Formación de ingenieros técnicos y dirigentes, capaces de plantear y dirigir técnicamente y de orientar la industria nacional.

\section{Antonio García Nossa: el visionario del Colegio Municipal, del Departamento Politécnico y la primera piedra de la Universidad}

En octubre de 1947se realizaron las elecciones para el Concejo de la ciudad, las cuales ganó ampliamente el partido liberal, que eligió 11 concejales sobre 4 del partido conservador. Entre los concejales elegidos se encontraba el economista bogotano Antonio García Nossa.

García Nossa había nacido el 16 de abril de 1912 y, aunque estudió Derecho en la Universidad Nacional, terminó sus estudios en la Universidad del Cauca en Popayán, donde además trabajó como profesor. Allí inició el Movimiento Indigenista Colombiano, motivo por el cual viajó en 1939 a México. En Bogotá creó un grupo compuesto por obreros, estudiantes e intelectuales que se llamó Liga de la Acción Política y empezó a publicar la revista Masas, que se distribuía entre los estudiantes de la Escuela Normal. En 1944, a la edad de 31 años, fue llamado por Gerardo Molina y junto a él fundó el Instituto de Economía que con el tiempo se convirtió en la Facultad de Economía de la Universidad Nacional. Fue asesor de Jorge Eliécer Gaitán y concejal de Bogotá entre 1947 y 1949.

Uno de los detalles más desconocidos de la vida de Antonio García Nossa tiene que ver con el proyecto que presentó cuando fue concejal de Bogotá en 1947, y cuyos resultados dieron origen a la hoy Universidad Distrital Francisco José de Caldas. En él descansó el proyecto de creación del Colegio Municipal de Bogotá. Fue García Nossa quien radicó y firmó el proyecto de acuerdo para la creación del Colegio Municipal de Bogotá, el 6 de diciembre de 1947. Este fue debatido de manera rápida pues ya en el mes de enero de 1948 se habían dado los dos debates para su aprobación. Ocho concejales de filiación liberal dieron el primer debate el 27 de enero y fue presentado como "Proyecto de Acuerdo número 12 de 1948 por el cual se crea el Colegio Municipal y se adoptan normas para su funcionamiento" (Anales del Concejo, 1948, 27 de enero, p. 135).En este primer debate se exponen varios artículos que denotan el tipo de institución planteada y la gratuidad de los estudios 
en ella ofrecidos así como el ánimo de apoyar a los sectores más pobres de la ciudad:

Artículo 1. Créase un Colegio de enseñanza segundaria para varones, que se denominará Colegio Municipal de Bogotá y funcionará como servicio gratuito para las clases trabajadoras. Artículo 2. El Colegio se organizará como externado, impartirá enseñanza gratuita, según los planes oficiales, y no se establecerá discriminación social alguna de familia, religiosa, partidista o económica. Artículo 3. La selección de los alumnos se hará por riguroso examen de concurso, prefiriendo a los niños egresados de las escuelas primarias oficiales y a quienes, poseyendo las capacidades intelectuales adecuadas, comprueben carecer de recursos económicos para costearse su educación. En igualdad de condiciones se preferirá a los hijos de los trabajadores municipales, empleados u obreros.

Se propuso que la partida para instalación del Colegio fuera de $\$ 50.000$, así como otras disposiciones donde aparece una primera organización del mismo. En la exposición de motivos quedó plasmado el espíritu de creación del Colegio, se expresaron las razones y motivos y se dio cuenta del estado de la educación en la ciudad. En conjunto, es una radiografía de lo que ocurría por aquella época y que García Nossa sustentó de la siguiente forma:

El presente año ha venido a demostrar que uno de los graves problemas sociales del Municipio de Bogotá es el referente a la falta casi absoluta de institutos de enseñanza secundaria para las clases trabajadoras. Las encuestas realizadas en los últimos días llevan a la conclusión bien dramática desde el punto de vista de las posibilidades de cultura de los hijos de empleados y obreros, de que solo en los tres colegios oficiales de enseñanza que funcionan en Bogotá desde hace 10 años, han sido rechazados más de 2.000 muchachos por falta de cupo. De otra parte, los colegios privados no solo tienen colmada su capacidad sino que, muchos de ellos, sin ofrecer una enseñanza técnica aceptable, están fuera de la capacidad económica de la totalidad de los empleados y obreros, ya que el gasto promedio por alumno en dichos colegios se calcula en $\$ 400$ anuales, sin computar los extraordinarios que ya han adquirido carta de naturaleza en tales instituciones inspiradas en concepto de lucro. En estas condiciones, el Concejo de Bogotá está en la obligación de intentar, en los términos de sus modestos recursos financieros, una mínima e inmediata solución al urgente problema. El Colegio Municipal de Bogotá que se persigue crear por medio del proyecto de Acuerdo que acompañamos, podrá atender a cerca de 300 alumnos -hijos de trabajadores municipales preferentemente, divididos en siete grupos: uno correspondiente al año preparatorio, dos al año tercero. Solo en 1951, estará el Colegio funcionando en la totalidad de los cursos de bachillerato. Y mientras en los colegios particulares el gasto medio anual por alumno es de $\$ 400$, el gasto anual para los padres en el Colegio Municipal no pasará de \$20 o \$25 (Anales del Concejo, 1948, número 1525).

Finalmente, mediante el Acuerdo 10 de febrero de 1948 se creó el Colegio Municipal de Varones. Antonio García Nossa entró a formar parte del consejo directivo del colegio, cuya rectoría quedó encargada al reconocido pedagogo Gabriel Anzola Gómez, quien años antes había editado los libros Orientaciones sobre Segunda Enseñanza (1939), Lecciones de Lógica y Teoría del Conocimiento (1945) y El Método Aplicado a la Enseñanza Primaria (1946).Estas y otras publicaciones sirvieron como experiencia para la designación de dicho cargo.

En1948, luego del asesinato de Gaitán, se abrió el colegio en el mes de julio. Ese mismo mes, Antonio García propuso ante el Concejo la creación de una Ciudad Politécnica, a partir del Departamento Técnico. El Colegio Municipal se convirtió en el cuarto colegio público de la ciudad y el primero del orden municipal. Además, como se dijo antes, el Colegio Municipal fue el único en crear un Departamento Técnico y el primero de su clase en ser fundado por la administración de la ciudad, lo que convierte a la hoy también IED Jorge Eliécer 
Gaitán en la institución educativa distrital más antigua.

Luego de los hechos del 9 de abril, se le cambió el nombre por el de Colegio Municipal Jorge Eliécer Gaitán. El día de la fundación, el diario El Tiempo reprodujo las palabras del primer rector del colegio, Gabriel Anzola Gómez:

Con íntima y sincera satisfacción asisto a este acto solemne, por medio del cual el municipio de Bogotá, inaugura el Colegio Municipal Jorge Eliécer Gaitán que fundó el H. Concejo por oportuna y feliz iniciativa del ilustre cabildante doctor Antonio García. De aquí que la creación de este Colegio Municipal, aprestigiado con el nombre del glorioso mártir de la democracia, venga a complementar aquel ciclo de adelanto y superación. ("Espléndida realidad es el Colegio Municipal Jorge Eliécer Gaitán”. El Tiempo, lunes 5 de julio de 1948, p. 3).

La presentación del proyecto de Acuerdo 107 de 1948 para la creación del Departamento Técnico del Colegio Municipal fue expuesta en los Anales del Concejo de Bogotá así:

El C. García explica que la Ciudad Politécnica no tendrá una orientación educacional como la Universidad, sino meramente técnica. Agrega que si se aplaza el estudio del proyecto podría quedarse indefinidamente y él aspira a que la Ciudad Politécnica sea una realización de este concejo. (Acta 56, Anales del Concejo, 6 de julio de 1949, p. 392).

Antonio García Nossa tuvo mucho que ver también con las primeras carreras de la futura institución universitaria. Además tenía claro el tipo de formación que esta seguiría durante sus primeros años:

Hay una suma orgánica de institutos y nos encontramos con una suma de construcciones que responde a unas cuantas fundamentales, cuales son el colegio, que no es una institución aislada de bachillerato sino que es un bachillerato eminentemente técnico, o sea que se orienta a dar la formación general y fundamental para seguir las carreras cortas auxiliares que o pueden hacerse dentro de la Universidad. En seguida se tiene la sección politécnica, o en las profesiones técnicas auxiliares superiores, tales como la de topógrafo, ayudante de laboratorio, dibujante industrial, estadígrafo, administrador, etc. (Anales del Concejo de Bogotá, agosto de1949).

Durante la permanencia de Antonio García Nossa en el Concejo de Bogotá, se enfrentó a quienes veían en el colegio una carga económica para el municipio. No obstante, García logró la adjudicación del lote en el barrio Modelo Norte, por ese entonces alejado del resto de la ciudad, la construcción de las primeras aulas y la adecuación de dos de ellas para el Departamento Técnico. La oficina de la secretaría del colegio funcionó en la calle $6 \mathrm{~A}$ número 43- 23. En la publicidad de la época se resalta el Curso Teórico de Topografía y el hecho de que el mismo no tiene costo alguno.

\section{El presbítero Daniel de Cayzedo y la Universidad Municipal}

En 1949 se realizaron los comicios electorales en todo el país. El partido liberal ordenó a sus candidatos abstenerse de presentarse en lista alguna, como una forma de protesta ante la violencia partidista desatada en los campos. Por tanto, el Concejo de Bogotá terminó siendo elegido en su totalidad bajo las banderas del partido conservador. Como había sido costumbre, se designaron funcionarios del partido en el poder, en las distintas dependencias municipales. El Colegio Municipal no fue la excepción. En remplazo de Gabriel Anzola Gómez fue nombrado el presbítero Daniel de Cayzedo, como nuevo rector, en el mes de marzo de 1950.

Daniel de Cayzedo fue un sacerdote jesuita nacido en Popayán. Doctor en Filosofía y Letras, estaba escalafonado en la primera categoría como profesor de Ciencias Biológicas por el Ministerio de Educación. Era un intelectual conocido en la ciudad ya que había participado de las reformas y métodos pedagógicos en los años cuarenta, asesorando al municipio en estos campos. En 1944 había publicado el libro Gramática y Antologías Latinas: 
Morfología y Sintaxis correspondientes al quinto y sexto curso de bachillerato según el programa oficial, por la editorial Voluntad. También publicó en 1955 El origen de las ideas en San Agustín y Aristóteles, además de una obra sin fecha de edición llamada Biblia, Filosofía y Siquiatría.

Desde su posesión, en el mes de marzo de 1950, Cayzedo manifestó su disposición por hacer que el Colegio Municipal y el Departamento Politécnico continuaran. Ante la ola de fundaciones universitarias en el país, Cayzedo consideró que era necesario ir más allá del Departamento Politécnico propuesto por García Nossa, de tal manera que formuló en junio de 1950la propuesta para la fundación de una Universidad Municipal en Bogotá. Posiblemente Cayzedo pensó que con ello podría asegurar mayores recursos para el colegio y quizás mayor atención por parte de los cabildantes. Tal vez por eso, pese a su formación humanista, no consideró en ningún momento la creación posterior de carreras con estas características; todo lo contrario, mantuvo la visión de Antonio García Nossa frente a la educación técnica y la llevó un paso más adelante aprovechando la presencia de varios profesores recién llegados de Europa.

No obstante, y siendo consecuente con la nueva "hegemonía conservadora", el nombre de Antonio García Nossa así como el de Jorge Eliécer Gaitán desaparecieron por varios años de los registros del Colegio y de la Universidad. Es por ello que por unanimidad se aprueba la creación de la Universidad Municipal de Bogotá Francisco José de Caldas a través del acta de fundación del 6 de agosto de 1950. El acta menciona en primer momento el nombre de Francisco José de Caldas, prócer de la independencia y que tenía en común con Cayzedo su ciudad natal: Popayán. Además de esta coincidencia, Caldas en sí mismo representa una aparente neutralidad frente a los tiempos de agitación política, por tanto se puede pensar que esta pudo haber sido otra de las razones para que la Universidad llevara este nombre, aunque en 1946 se conmemoraron 130 años de su muerte y en 1948, 140 años de su nacimiento, por lo que incluso se editó una estampilla por tal motivo. Llama también la atención que la Universidad Municipal no fuera la única institución creada ese año, que ostentó el mismo nombre. El Instituto Nacional Francisco José de Caldas de Villavicencio fue creado en el mes de octubre. También se instauró la Medalla Militar Francisco José de Caldas mediante el Decreto 3404.

No obstante, los registros de la participación de Antonio García desaparecieron de la historia oficial de la Universidad y hasta hoy se dice que la iniciativa de la creación del Colegio Municipal de Bogotá y luego de la Universidad Municipal fue autoría del mencionado presbítero Cayzedo, que si bien es cierto materializó el proyecto de García Nossa, por otro lado, borró quizás muy a su pesar, o porque no significaba mucho para él, la participación del prominente economista en la historia de la institución.

La fundación de la Universidad fue descrita por el diario conservador El Siglo:

La Universidad Municipal de la Capital se fundará oficialmente esta tarde: tendrá especializaciones sobre carreras menores, con cinco facultades. El Acta se firma esta tarde. El decreto correspondiente será expedido hoy. La Universidad Municipal de Bogotá será inaugurada oficialmente hoy a las dos de la tarde en una ceremonia simbólica que se efectuará en el despacho del Alcalde Santiago Trujillo Gómez, con la asistencia de miembros de la junta directiva del Colegio Municipal y de otras personalidades invitadas especialmente. El acta de fundación será firmada por el Presidente Ospina Pérez, el Gobernador Jorge Leiva, el Alcalde Trujillo Gómez, el Ministro de Educación Mosquera Garcés, el Vicario Capitular de Bogotá, Monseñor Emilio de Brigard, el Vocal del Concejo Municipal Carlos Alberto Guzmán, el Vocal de la Universidad Nacional, Álvaro Marín, el Rector del Colegio, presbítero Daniel Cayzedo, el Inspector de Educación Municipal de Bogotá, Luís Eduardo Sánchez y el Secretario del Consejo Directivo del Colegio Municipal, Salvador Linares. La Universidad Municipal constará de cinco facultades de especializaciones menores y que en su orden serán: Ingeniería radio técnica, 3 años, ayudante de topografía, 2 años, perito forestal, 2 años, ayudante de geólogo, 3 años, y perito en sondeos y perforaciones de pozos, 2 años. La Universidad Municipal 
de Bogotá será la primera entidad educativa de su género que funciona en el país. Hasta el momento se habían hecho algunos intentos que prácticamente no culminaron en nada satisfactorio. Por su parte, La facultad de radio-técnica con estudios sistematizados será el primer plantel universitario que funcione en América del sur (El Siglo. 6 de agosto de 1950, pp. 1-7).

Finalmente, como aparece en el acta de fundación, fue el presidente conservador Mariano Ospina Pérez, en uno de sus últimos actos de gobierno, quien firmó el documento que dio vida a la Universidad Municipal, una entidad sin presupuesto, sin una sede, sin aprobación alguna, cuyo desarrollo quedó en manos del rector del colegio el presbítero Cayzedo. Ese mismo año, Cayzedo gestionó la resolución así como el trámite para su funcionamiento, lo cual merece todo el mérito, ya que no contó con recurso alguno salvo los que le permitió la junta directiva del colegio, como consta en las Actas del Concejo de Bogotá.

En octubre, el Ministerio de Justicia le otorgó la personería jurídica a través de la Resolución 139 de 1950 y por Decreto 88 de febrero 26 de 1952 la Alcaldía Municipal permitió la enseñanza de la Universidad, bajo el amparo legal de ser un instituto de enseñanza profesional. Con el Decreto 88 de febrero 26 de 1952 se dio reconocimiento a los estudios y títulos que la universidad proponía. Así, mediante la Resolución 403 de 1952 el Ministerio de Educación Nacional se concedió el permiso autorizando provisionalmente el funcionamiento de la Universidad Municipal como instituto de enseñanza profesional, en las ramas de Ingeniería Radio Técnica, Ingeniería Forestal y Topografía y otorgando el título de licenciado en el área respectiva. Finalizando ese año, se expidió el Decreto 653 de noviembre 13 de 1952, el cual amplió el alcance del Acuerdo 51 de 1948, en el sentido de crear carreras de larga duración que la habilitaron para otorgar títulos estrictamente académicos.

Finalmente, por medio de la Resolución 3410 de diciembre 9 de 1952 el Ministerio de Educación Nacional aprobó los estatutos de la Universidad en razón a encontrarse los programas de estudio de las facultades de Topografía, Ingeniería Forestal e Ingeniería Electrónica conformes a lo dispuesto en el artículo 12 de la Ley 56 de 1927 y el Decreto 260 de 1936. En la Resolución 5128 se encuentra la aprobación definitiva que a los planes de estudio concedió el MEN. La Universidad inició oficialmente clases en 1951, en 1953 tuvo sus primeros egresados; paradójicamente su graduación condujo a que en la práctica cerrara sus puertas debido a que no se hizo una nueva apertura de cupos y solo hasta febrero de 1955 volvió a abrir sus puertas, esta vez muy lejos del colegio que la vio nacer. Por su parte, el presbítero Cayzedo se retiró de la rectoría en 1954, dejando a cargo de la institución a Rafael Díaz Becerra, quien estuvo algunos meses en el cargo hasta comienzos de 1955, cuando asumió Jesús Casas Manrique.

\section{Discusión}

Teniendo en cuenta la existencia de una información oficial publicada en la página web de la Universidad Distrital Francisco José de Caldas, los resultados parciales de esta investigación permiten confrontar la información y establecer en algunos de sus apartes que:

Página web (PWUD):"La Universidad Distrital Francisco José de Caldas fue fundada en el año de 1948, por iniciativa del presbítero Daniel de Cayzedo, quien además fue su primer rector". La Universidad fue fundada en 1950. En 1948 se fundó el Colegio Municipal por iniciativa del economista Antonio García Nossa. El primer rector del colegio fue el pedagogo Gabriel Anzola Gómez. El presbítero Daniel de Cayzedo solo aparece en la escena cuando es nombrado por el Concejo de Bogotá en marzo de 1950. Por tanto el presbítero es el primer rector de la Universidad pero no del colegio. En todo caso, la iniciativa de creación de la Universidad es del presbítero, pero retomando los planteamientos de Antonio García Nossa.

Página web (PWUD):“La Universidad funcionó por primera vez donde hoy se encuentra el Museo de Desarrollo Urbano en la calle 10 con carrera 8a., frente al Palacio Liévano y el Capitolio Nacional, en lo que hoy se constituye como un sitio histórico de la ciudad". Los datos encontrados, además de la confrontación con las entrevistas de los primeros 
egresados, dan cuenta de que esto es falso. La primera sede fue la que ocupó el Colegio Municipal en el barrio Modelo Norte. Posteriormente se ocupó la Casa Navarro, actual sede del Instituto Pensar de la Universidad Javeriana.

Página web (PWUD): “A partir de este momento se pierde todo dato histórico de la Universidad hasta el año de 1957 cuando la Junta Militar de Gobierno de entonces le da el carácter de Universidad del Estado. En aquel entonces, la ciudad de Bogotá pasó a llamarse Distrito Especial y la Universidad se llamó Universidad Distrital". La afirmación "pérdida de todo dato histórico" no es correcta. Solo refleja la ausencia de trabajos históricos al respecto, los cuales forman parte de la investigación en desarrollo.

Página web (PWUD):“Todo parece indicar que el nombre de Francisco José de Caldas lo tomó la Universidad al momento en el cual se expidió el decreto de la Junta de Gobierno para cambiar el nombre de Universidad Municipal de Bogotá por el de Universidad Distrital Francisco José de Caldas". Falso. Desde el documento del Acta de Fundación del 6 de agosto de 1950 aparece el nombre de "Francisco José de Caldas". Esa apreciación corresponde a la falta de una lectura del mencionado documento. En todo caso, su nombre coincide con la ciudad de origen del presbítero Cayzedo: Popayán. Ese mismo año se crearon varias instituciones educativas y se instituyó la Medalla Militar con ese nombre.

\section{Conclusiones generales}

Se establece que el Colegio Municipal de Bogotá y su Departamento Politécnico nacen en medio del debate ante la crisis de las Escuelas Industriales. Representa su contrapartida y, de alguna manera, la fundación de la Universidad fue la forma adecuada para mantener la posibilidad de una educación accesible a los sectores populares de la ciudad. Al mismo tiempo se establece la importancia de la política nacional en el desarrollo de las instituciones colombianas. Un proyecto nacido de manos de un grupo liberal en 1947 tornó en 1950 hacia la fundación de la Universidad Municipal, como parte de un proyecto conservador. Fundada oficialmente bajo la firma del presidente conservador Mariano Ospina Pérez como consta en el acta de fundación, se convirtió durante esa década en proyecto de ese partido de manera breve hasta la intervención de Rojas Pinilla. Sus antecedentes liberales en la práctica se desconocieron al punto que hoy no se menciona la presencia de Gabriel Anzola como primer rector ni la de Antonio García Nossa como artífice del colegio.

Agradecimientos: Centro de Documentación Lebecs, Universidad Distrital, Library of Congress (EEUU), Oficina de Egresados Universidad Distrital, Instituto de Estudios e Investigaciones Educativas (IEIE) Universidad Distrital.

\section{Referencias}

Álvarez, M. (2002).La formación de ingenieros y el problema de la modernización colombiana 1904-1991. Pasto: Universidad de Nariño.

Álzate, J. \& Parra, R. (1976). Los determinantes de la escolaridad. Aportes para el análisis de la educación urbana en Colombia. Bogotá: CEDE, Universidad de los Andes.

Anzola Gómez, G. (1976). Sobre la Universidad. Aspectos académicos. Bogotá: Universidad Externado de Colombia

Archila, M. (1999). Entre la academia y la política: el movimiento estudiantil en Colombia, 19201974. En: R. Marsiske \& L. Alvarado, Movimientos estudiantiles en la historia de América Latina (pp. 158-174). Ciudad de México: Universidad Nacional Autónoma México.

Arciniegas, G. (1994). Cuadernos de un estudiante americano. Bogotá: Ediciones Uniandes, Educar.

Barriga, M. (2004). La educación musical en Bogotá. 1880-1920. Revista de Investigaciones en Música y Artes Plásticas, 1, Universidad de Pamplona.

Becerra, A. \& Restrepo, O. (1993). Las ciencias en Colombia: 1793-1990. Una perspectiva histó- 
rica sociológica. Revista Colombiana de Educación, 26, Bogotá.

Betancourt, P. (1914, enero). Artículo 17 de la Ordenanza 30 de 1913. Revista Instrucción Antioqueña (p. 282), Imprenta Departamental de Antioquia.

Burle, P. (2000). Formas de historia cultural. Madrid: Alianza.

Cabrera, M. A. (2001). Historia, lenguaje y teoría de la sociedad. Madrid: Fronesis Cátedra Universitat de Valencia.

Cáceres, H. (1951, noviembre 17). Problemas de la Educación colombiana: El Destino de la Juventud. La Nación, Bogotá, p. 4.

Cajiao, A. (1952). El Impuesto de valorización. Trabajo presentado por el ingeniero Abelardo Cajiao V., en el ciclo de conferencias de información técnica auspiciada por la Universidad Municipal Francisco José de Caldas. Anales de Ingeniería, 57(633), Bogotá.

Díaz, J. (2010). El Movimiento Obrero Estudiantil Campesino 7 de Enero y los orígenes de la nueva Izquierda en Colombia. 1959-1969. Tesis de Maestría en Historia. Bogotá: Universidad Nacional de Colombia.

Flórez, C. (2000). Las transformaciones sociodemográficas en Colombia durante el siglo XX. Bogotá: Banco de la República y Tercer Mundo.

Gaitán, J. (1940). Presentación del informe de educación a las cámaras legislativas de 1940. En: J. E. Gaitán, Obras Selectas. Colección Pensadores Políticos Colombianos, Cámara de Representantes. Bogotá: Imprenta Nacional.

Galasso, G. (2005). Nada más que historia. Teoría y metodología. Barcelona: Ariel Historia.

García, A. (1978). Planificación municipal. Bogotá: Universidad Distrital Francisco José de Caldas.
García, A. (1985). La crisis de la universidad. La universidad en el proceso de la sociedad colombiana. Bogotá: Plaza \& Janés.

González, G. R. (1992). La experiencia colombiana en materia de evaluación de la educación superior. En: Evaluación, promoción de la calidad y financiamiento de la educación superior (pp. 55-57). México: SEP-Conaeva.

Henderson, J. (2006). La modernización en Colombia. Los años de Laureano Gómez, 1899-1965. La educación de los centenaristas. Medellín: Universidad Nacional de Colombia.

Herrera, M. (1993). Historia de la educación en Colombia. La República Liberal y la modernización de la educación: 1930-1946. Revista Colombiana de Educación, 26, 107.

Icfes. (1977). Historia estadística de la educación superior colombiana. Bogotá.

Jaramillo, J. (1978). El proceso de la educación del virreinato a la época contemporánea. En: $M a$ nual de historia de Colombia. Tomo III. Bogotá: Colcultura.

Jiménez, C. \& Guevara, R. (2002). La Universidad como proyecto. Bogotá: Universidad Distrital.

Lobo, L. (1988). La producción académica de los estudiantes de Ciencias Sociales 1975-1988. Tesis de grado. Bogotá: Universidad Distrital.

Luzuriaga, L. (1978). Historia de la educación y de la pedagogía. Buenos Aires: Lozada.

Martínez, C. (2008). En pos de la memoria. Entrevista a tres de los primeros egresados de la Universidad Distrital. En: Universidad Distrital: sesenta años de memoria y vida. Bogotá: Universidad Distrital.

Martínez, C. (1937). Escritos Políticos. Vol. 8. G. Otero y L. Martínez (eds.). Bogotá: Imprenta Nacional. 
Melo, J. (1986, marzo). La ciencia en Colombia en el siglo XX. Revista de la Universidad de Antioquia, 4(19). Medellín.

Moreno, B. (1949). Gaitán ante sí mismo. Entrevista realizada por B. Moreno Torralba. El Siglo, julio 1943. En: Documentos para una biografía. Registro Municipal. Bogotá: Imprenta Municipal.

Nieto, A. (1915). Las cajas escolares y su acción en Bogotá. Con prólogo de Tomás Rueda Vargas. Bogotá: Arboleda y Valencia Editores.

Palacios, J. (1985). La cuestión escolar. Barcelona: Laia.

Parra, S. (1996). Escuela y modernidad en Colombia. Tomo IV: La Universidad. Santa Fe de Bogotá: Tercer Mundo.

Pécaut, D. (1987). Orden y violencia en Colombia. Bogotá: Siglo XXI, 2 vols.

Reina, C. (2008). El contexto de creación de la Universidad Distrital "Francisco José de Caldas". En: F. Santamaría, Universidad Distrital, 60 años de memoria y vida. Bogotá: Universidad Distrital.

Rojas, J. (1988). Historia de la educación en Colombia. Bogotá: Ecoe.

Santamaría, F. (2008). Universidad Distrital, 60 años de memoria y vida. Bogotá: Universidad Distrital.

Silva, R. (2005). República Liberal, intelectuales y cultura popular. Bogotá: La Carreta Histórica.

Silva, R. (1989). La educación en Colombia. 18801930. En: Nueva historia de Colombia (vol. IV, p. 75). Bogotá: Planeta.

Soto, D. (1988). Historia de la universidad colombiana. Historiografía y fuentes. Tunja: UPTC.

Soto, D. (1997). Historia de la universidad colombiana. Resúmenes analíticos. Tunja: UPTC.
Soto, D. (2004). Historia de la universidad latinoamericana. De la colonia al siglo XXI. España: Doce Calles.

Soto, D. (2006). Aproximación histórica de la universidad colombiana. Tunja: UPTC.

Tirado, A. (1986) (comp.). 50 años de la reforma de 1936. Bogotá: Contraloría General de la República.

Tirado, A. (1988). Aspectos políticos y sociales de las reformas de Alfonso López Pumarejo (19341938). Bogotá: Procultura.

Tovar, B. (1984). La intervención económica del Estado en Colombia 1914-1936. Bogotá: Banco de la República.

Tunnermann, B. (1997). Aproximación histórica a la universidad y su problemática actual. Santa Fe de Bogotá: Universidad de los Andes.

Universidad Nacional. (2001). Gerardo Molina y la Universidad Nacional de Colombia. Bogotá: Universidad Nacional.

Weber, M. (1995). El político y el científico. Barcelona: Altaya.

Zalamea, E. (1953, abril 26). La generación que se asomó al poder. Dominical, p. 5.

\section{Otros documentos}

Ciclo de conferencias sobre asuntos administrativos bajo los auspicios de la Universidad Municipal de Bogotá Francisco José de Caldas. Bogotá: Imprenta Municipal, 1952.

El Siglo (1940, 1942, 1948, 1950, 1951).

El Tiempo (1940, 1947, 1949, 1950, 1951).

Anales del Concejo de Bogotá (1947, 1948, 1949, 1950, 1951, 1952).

Historia de Bogotá. Tomo II. Siglo XX. Bogotá: Salvat y Villegas, 1989. 
Historia del Instituto Técnico Central. Fuente: www. itc.edu.co

Censo General de Población 5 de julio de 1938. Ordenado por la Ley 67 de 1917. Resumen general del país. Contraloría General de la República. Dirección Nacional de Estadística. Bogotá: Imprenta Nacional, 1942.

Página web www.udistrital.edu.co
Agradecimientos: artículo de revisión derivado del proyecto de investigación "Historia de la Facultad de Ingeniería de la Universidad Distrital Francisco José de Caldas: constitución de un archivo histórico y documental", aprobada y financiada por el Centro de Investigación y Desarrollo Científico (CIDC) de la Universidad Distrital, y enmarcado dentro de los proyectos apoyados por el Instituto de Estudios e Investigaciones Educativas de la Universidad Distrital Francisco José de Caldas (IEIE). 